\title{
The I-beam steel temperature field analysis in cooling control process after hot rolling
}

\author{
Zhan WANG ${ }^{1,2 a}$, Fenglong ZHU ${ }^{1,2 b}, \mathrm{Ju} \mathrm{WANG}^{3}$, Haibo WANG ${ }^{3}$ \\ ${ }^{1}$ School of Mechanical Engineering, Shenyang jianzhu University, Shenyang 110168, China \\ ${ }^{2}$ National-local joint engineering laboratory of high-grade stone, NC processing equipment and \\ technology, Shenyang jianzhu University, Shenyang, 110168, China \\ ${ }^{3}$ Shenyang machine tool(Group)Co.,Ltd, Shenyang 110000, China \\ aemail: juven1126@163.com, bemail:2215183918@qq.com
}

Keywords: I-beam Steel; Cooling Control; Instantaneous Temperature Field; Numerical Simulation; Finite Element Theory

\begin{abstract}
Basing on the heat transfer theory, the process of Q235 I-beam steel which is chosen to be studied could be done after rolling cooling control analysis. Given the practical manufacturing conditions, the finite element theory and its temperature field can be built to control the cooling process of I-beam steel hot rolling. There are three projects that can adopted to do the numerical simulation analysis in controlling the cooling process for its each part temperature distribution, meanwhile the temperature decline curves and instantaneous temperature field distribution could be achieved under different conditions such as cooling time, cooling velocity, ending cool temperature and the major factor that affects the temperature field distribution can also be find. The feasibility of this project has been proved by comparing and analyzing the theoretical results and its experimental outcomes to the cooling control process, so that it can be consulted to design the cooling control process reasonably.
\end{abstract}

\section{Introduction}

With the establishment of designing discipline to the I-beam steel hot rolling, it has been widely used in industry. Users have an improving requirements to I-beam properties, especially to large scale projects, high-rise buildings. It has become a tough question on how to compete with foreign high-performance products and how to improve the high-performance economically to our national I-beam enterprises[1]. So the research is focusing on the hot rolling process and cooling control methods. After consulting articles and documents about I-beam hot rolling cooling control process[2-4], it can be concluded that it not only improves the mechanical properties, but also decreases the residual stress and nonuniform deformation[5-7]. So the promotion of cooling control process plays an important role in improving rolling qualities of the steel, however at the original stage of rolling cooling control, its surface temperature picks up because of the relative slow cooling velocity in internal and will finally tend to be uniform with its internal temperature. It can be concluded that the temperature field changes are complicated during the rolling process, so the modeling to its temperature field is necessary and the optimal disguised project can be gotten through analysis to the I-beam hot rolling. The purpose of calculating the temperature field are as follows:

(1) It can be taken as a calculating method of the rolled steel temperature field in cooling control, which is an important parameter in cooling control process.

(2) The rolled steel after cooling return temperature can be confirmed after calculating the temperature field according to the before the cooling temperature and the condition, or it can provide reference for the cooling process of its demands for the return temperature.

(3) The internal temperature stress will be caused in rolled steel fracture section when cooling. If too much temperature stresses, cracks can be generated.

(4) The phase transition structure of the rolled steel fracture section can be ensured through 
combining cooling curves and material continuous cooling changes curves which are results of calculating rolled steel field on its sections, therefore its relativity can also be judged.

\section{Temperature gradient finite element modeling}

The study bases on parametric design language as a tool for modeling .It is very important to determine the unit type before starting the finite element analysis. Under normal circumstance, comparing with a liner unit using the second order unit will get a higher accurate result by adopting lass units and freedom degree, meanwhile given the modeling joist steel surface boundary will have a complicated heat exchange because of water-cooling, the second order unit is a more accurate choice to describe curve boundary or hook face .

ANSYS software has supplied a division tool which assists users with many kinds of grid division so that they can match the division tool with model shapes and unit character. Actually,it is not to choose division tool in accordance with shape classification but to ensure the unit shapes after choosing division tool.The article has adopted the proportional line segment freedom control grid division method The purpose of manually control unit density is just to care about the unit density, that is to say only through adopting a unit size that conforms dense to sparse grid form from the section boundary line to its inner normal direction of a joist steel, can it be guaranteed to get a right outcome.

The simulation discretizes the grid by means of plane 77 which is a eight nodes, two dimension and two order unit As to the two dimension simulation joist steel, proportional line segment freedom control grid division should be taken to divide it into 998 units and 1093 nodes After calculating, this division method could be satisfied both in accuracy and fast calculation. The two dimension finite element model of I-beam is selected as a specimen ,as shown in Fig. 1.

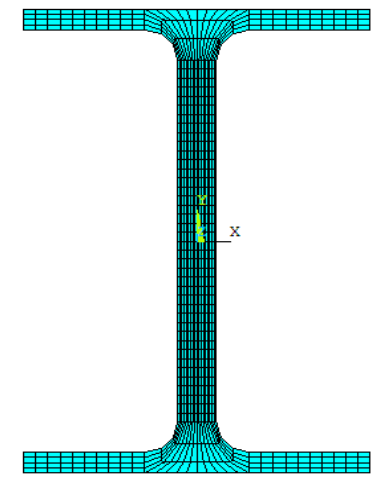

Figure 1. The two dimension finite element model of I-beam

The cooling control after the hot rolling I-beam is the instantly nonlinearity analysis, which the original temperature and convection current should be defined respectively as the primary and boundary premise.

(1) There is a natural convection heat exchange between the hot rolling I-beam and its outside air.

(2) There is a forced convection heat exchange between the water cooling duration of the hot rolling I-beam and cool water.

Given the three procedures of air cooling, water cooling, and air cooling of the I-beam steel cooling control, circulatory applied load method should be taken firstly. Next,confirm the cooling time span and time step-size .After calculating, cooling time is 5 seconds and the time step-size that from the shortest $0.01 \mathrm{~s}$ to the longest $1 \mathrm{~s}$ is 0.05 seconds. Load step choice is defined as a specimen, as shown in table. 1.

\begin{tabular}{|c|c|c|c|c|}
\hline step & $\begin{array}{l}\text { Way } \\
\text { cool }\end{array}$ & T-step & Max step & Min step \\
\hline 1 & Air & 5 & 0.5 & 05 \\
\hline 2 & Water & 10 & 0.2 & 0.2 \\
\hline
\end{tabular}




\section{Numerical simulation of temperature field cooling control}

During the cooling control process, given the almost equal cooling condition ,it is inevitable to generate nonuniform temperature distribution in cross section because of different part of I-beam sizes .Faced with this situation, do some cooling control is a must. In the process of cooling, it is also inevitable to produce discrimination both in temperature and deformation along the cross section and will cause the nonuniform properties of the I-beam in the end, because of its complicated cross section.

After modeling I-beam rolling, it is a common practice to do the last air cooling procedure. So it can be approximately combined convection heat exchange with radiation heat exchange under the comprehensive consideration with respect to the low convection heat exchange coefficient and its small fluctuation range. In this study, taking the calculation method of empirical formula and comprehensive heat exchange coefficient into consideration, the environmental temperature can be set by 20 centigrade.

After the process of air cooling, water cooling and air cooling, the temperature changes on the surface of I-beam cross section is fiercely, while they are stable on the center section. The I-beam has a little temperature reduction which decreases only 20 centigrade in five minutes before water cooling. when water cooling, it will have a fierce changes which decreases to about 500 centigrade because of the fast heat exchange. when it comes to tempering, the section temperature will pick up with the delivery of center heat and tend to accordance finally. In order to investigate the temperature changes discipline in different part of the I-beam during the cooling control, there are three measures can be taken. The first one is 5 seconds air cooling following with 10 seconds water cooling and 5 seconds tempering. The second one is 5 seconds air cooling following with 15 seconds water cooling and 5 seconds tempering. The third one is 5 seconds air cooling following with 8 seconds water cooling and 5 seconds tempering.

The first project is 5 seconds air cooling following with 10 seconds water cooling and 5 seconds tempering from which can be discovered a temperature gradient distribution discipline by 10 seconds numerical simulation in water cooling stage and 20 seconds to the whole stages.10 seconds temperature gradient distribution of the I-beam in water cooling is selected as a specimen, as shown in Fig. 2(a). The whole 20 seconds stages of temperature gradient distribution is selected as a specimen, as shown in Fig. 2(b).As can be seen from the picture, temperature range is from 576.265 centigrade to 653.565 centigrade, The lowest grade is 575.265 centigrade which distributes at the joint section between loin and beam. At the two edges of the beam, the fluctuation of the loin is a gradual increase from 627.464 to 653.563 , when it comes to water cooling stage, there is a sharp plunge on the fracture section, especially the node at the far-end angle part. After cooling, there is a temperature rising phenomenon at the node between the beam and loin joint because of the slight high temperature at the node of geometric center and its diffusion to surface in twice air cooling process. During the whole cooling control process, the biggest temperature surplus of those four different parts node occurs at the end of water cooling

In the first project, there are node temperature decline curves from TEMP-2 to TEMP-5 representing different parts of the I-beam, among which are respectively waist geometric center, beam geometric center, rounded angle on beam and waist joints, the far-end angle beam part.As can be seen from the Fig.2(c).

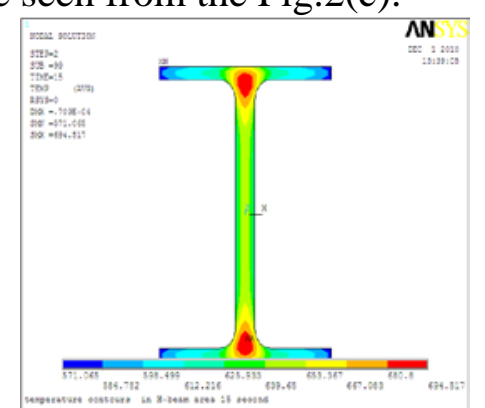

(a) 10 seconds water cooling

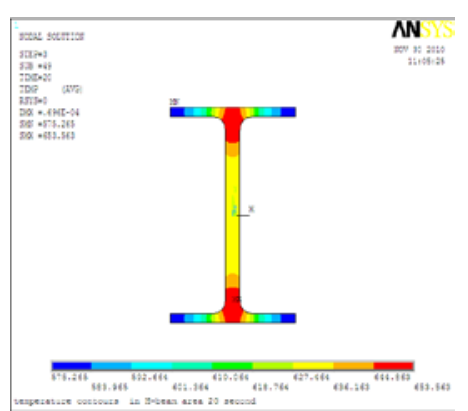

(b) 20 seconds water cooling

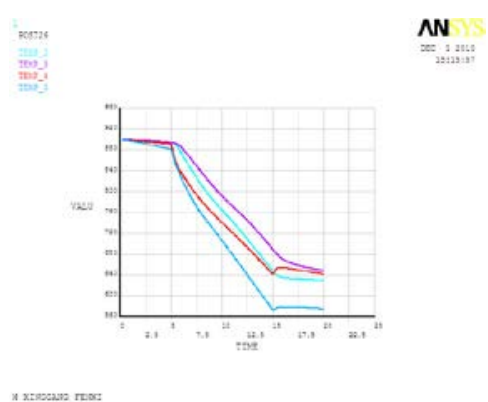

(c) temperature decline curves

Figure 2.Temperature field distribution in cooling process 
The second project is 5 seconds air cooling following with 15 seconds water cooling and 5 seconds tempering. The discipline of temperature gradient distribution can be discovered in the project through the 15 seconds numerical simulation to water cooling stage and 25 seconds to the whole stages .As can be seen from Fig.3(a). and Fig.3(b). The temperature fluctuation is from 585.487centigrade to 624.278 centigrade, which are respectively distributing at the edge of the waist center and beam, within the connection zone. The middle waist part temperature is 585.487 and fluctuation is a gradual increase from the center to two edges.

In the second project, node temperature decline curves on different parts of I-beam can be seen from the Fig.3(c). During the air cooling, there are hardly any changes. When getting into water cooling stage, comparing with the first project, there is a slow temperature change on the fracture section of I-beam. The temperature surplus of nodes among different parts in the whole cooling control process is a little small and the biggest temperature gap of node on four different parts is about 60 centigrade.

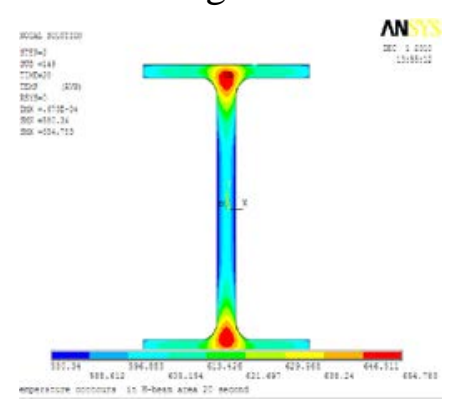

(a) 15 seconds water cooling

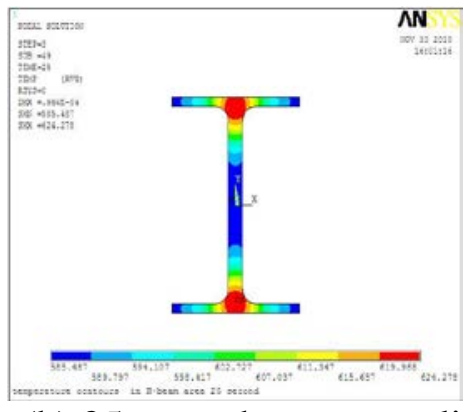

(b) 25 seconds water cooling

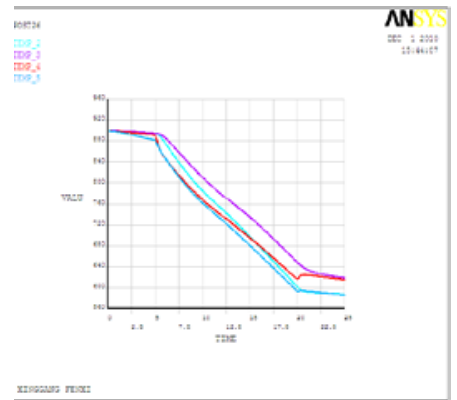

(c) temperature decline curves

Figure 3.Temperature field distribution in cooling process

The third project is 5 seconds air cooling following with 8 seconds water cooling and 5 seconds tempering. The discipline of temperature gradient distribution can be discovered in the project through the 8 seconds numerical simulation to water cooling stage and 18 seconds to the whole stages. As can be seen from Fig.4(a). and Fig.4(b). The temperature fluctuation is from 611.822 centigrade to 688.321 centigrade, which are respectively distributing at the edge of the waist center and beam, within the connection zone. The waist part temperature is 622.821centigrade and the fluctuation is a gradual increase from the waist center to two edges. As can be seen from Fig.4(c).

In the third project, node temperature decline curves on different parts of I-beam can be seen from the Fig.4(c). The temperature decrease ratio is higher and the temperature surplus of nodes among different parts in the whole cooling control process is more higher and the biggest temperature gap of node on four different parts is about 140 centigrade. Considering the high cooling rate and bigger internal temperature gap ,it will do harm to the control of I-beam deformation. So it should be avoided much higher cooling rate and temperature surplus.

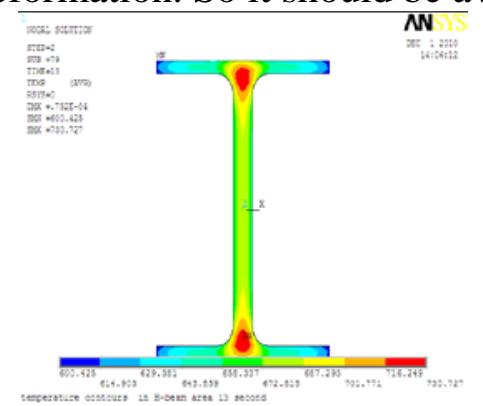

(a) 8 seconds water cooling

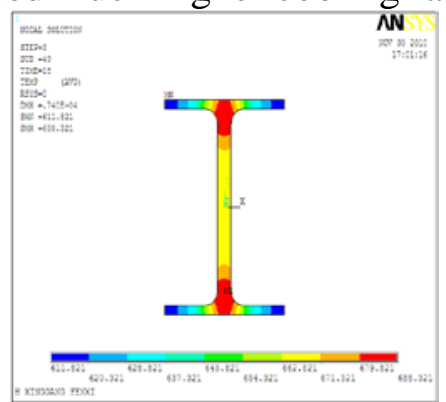

(b) 18 seconds water cooling

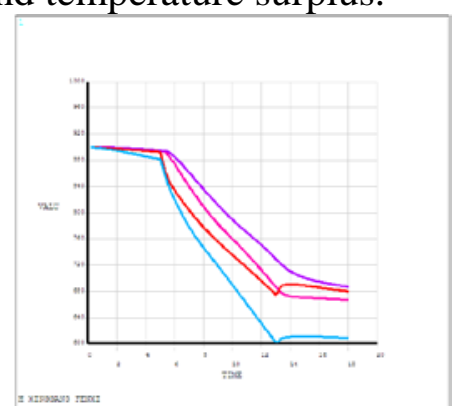

(c) temperature decline curves

Figure 4.Temperature field distribution in cooling process

It can be concluded from above three projects numerical simulation and temperature curves, as followings:

(1) Cooling control has improved cooling velocity than natural cooling, but the temperature gap is also increased on the different surfaces of I-beam, which enlarges the temperature nonuniform.

(2) There are complicated temperature distribution disciplines in the I-beam web and nodes on beam fracture section in those three projects, among which the common ground is that the highest 
temperature spots at the joint of the middle beam edge and the web, the differences are respectively the lowest temperature at the middle web and beam ends in the second project and the higher temperature that exists about 51 centigrade surplus of the web middle than the beam ends in the first and third project. It will have a fast cooling velocity and low temperature because of the strong water cooling strength and the fast heat dissipation, however there exists a higher temperature at the joint of the middle beam and web because of the weaker water cooling strength,the slow heat dissipation, and the transitional fillet.

(3) At the initial cooling stage, the web and the beam will experience a fast cooling, temperature gradient enlarging and the temperature increase in fracture sections under the circumstance of high temperature, strong heat radiation rate and a relatively stable heat convection exchange premise. With the decline of the temperature, it will have a heat radiation rate downturn, slow temperature, temperature gradient narrowly and the temperature gap plunging in all parts.Considering the bigger temperature gap causes overlarge interior stress and the deformation to the I-beam, so it must to be done especially to those big size I-beam that under the processing premises to control the cooling time and decrease the temperature gap including the I-beam surface and center and its thermal difference between top and bottom surface. Temperature nonuniform is also a primary factor to contribute to the after cooling residual stress, which is an important study in cooling control technical research.

(4) Comparing those three projects, it can be inferred that the second project possesses the priority in respect of temperature controlling, temperature gap and avoidance in fast cooling rate than those two. Overall, the second project has a better cooling effect and it is the optimal project.

(5) It is because of the cooling uniformity has a direct influence on the inner organization field uniformity that it stands a significant position in cooling control velocity to decrease the temperature gap between surfaces and interior of the I-beam.

\section{I-beam air cooling temperature field model testing}

In order to test the correctness of finite element model analysis, a I-beam with $100 \mathrm{~mm} \times 65 \mathrm{~mm}$ can be tested on the cooling control experimental device. As can be seen from Fig.5, they are respectively small size heater and roller bed cooling device. There is a temperature measurement before and after the cooling control, which are optical pyrometer and infrared thermodetector and the measuring position spots at the half I-beam edge breadth. It can be tested to the mechanical property after cooling control.The error and accuracy of experiment results could be tested by way of combining the temperature field changes character and the verification -comparison and error analysis which are achieved through numerical modeling and experiment results approximately in accordance with the experiment results whose error range is from $6.57 \%$ to $9.61 \%$ and the biggest error is $15.06 \%$ if not cooling.

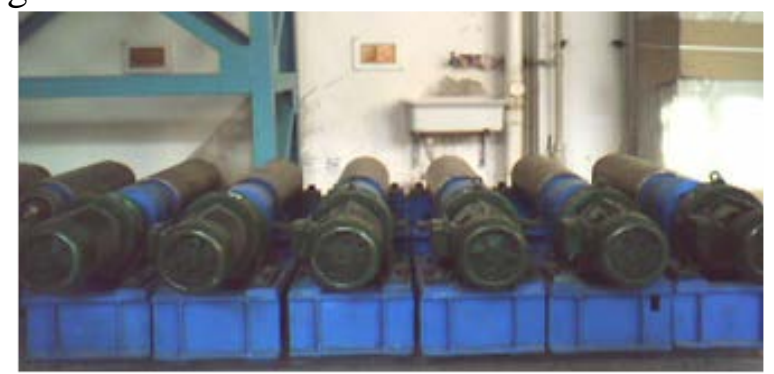

Figure 5. Roller bed cooling control device

\section{Conclusions}

In this article, the temperature field model is analyzed through building up the two dimensions model and the temperature field model for the I-beam steel and adopting three cooling control projects. The temperature field varying characters and its disciplines have been discovered and also its temperature decline curves in different four parts. The second cooling control project is ensured 
by analyzing those three and it has passed the experimental testing that the outcome is close to the modeling result and the error is between $6.57 \%$ to $9.61 \%$. In this article, the outcome has proved its feasibility and correctness of the finite element analysis method to temperature field in cooling control after rolling so that it could on the one hand provide references for the cooling control design and one the other hand provide a method of guidance for mastering the I-beam steel cooling disciplines and developing a new cooling control process.

\section{Acknowledgement}

In this paper, the research was sponsored by the Scientific Research Foundation of Shenyang Jianzhu University(Project No.2014011) and the Discipline Content Education Project of Shenyang Jianzhu University(Project No. XKHY2-33) and the Open Foundation of National-Local Joint Engineering Laboratory of NC Machining Equipment and Technology of High-Grade Stone(SJSC-2015-14) and

\section{References}

[1] Qian Chen. Advanced long profile rolling [J].Journal of Iron and Steel Research, 2005, 17(2):7-11.

[2] Dejun Su, Wang Jin, Chunhui Zhao. Review on structural steel thermoplastic deformation after the cooling process of numerical simulation studies [J].Journal of Plasticity Engineering, 2005, 12(3):4-9.

[3] Yuan Guo, Zhaodong Wang, Guodong Wang. The application of cooling control in the plate strip development and production [J].Journal of Iron and Steel Research, 2006, 18(1):1-5.

[4] Bakkaloğlu A, Effect of processing parameters on the microstructure and properties of an $\mathrm{Nb}$ microalloyed steel, Materials Letters, 2002, 56(3):200-209.

[5]Nowotnik A, Siwecki T, The effect of TMCP parameters on the microstructure and mechanical properties of Ti - Nb microalloyed steel, Journal of Microscopy, 2010, 237(3):258 - 262.

[6] Hauru Wang, Aixia Wan, Yingwang Wang. High line wear water-cooling process numerical simulation of temperature field, Journal of YanShan University, 2008, 0129(6):476-479.

[7] Liu Cai, Zhenshan Cui. The plate hot rolling coupled thermo-mechanical finite element simulation [J].Journal of Mechanical Engineering, 1998, 34(4):35-39. 